\title{
Distribution-Free Continuous Review Inventory Model with Controllable Lead Time and Setup Cost in the Presence of a Service Level Constraint
}

\author{
Wei-Min MA and Bing-Bing QIU \\ School of Economics and Management, Tongji University, Shanghai 200092, China \\ Correspondence should be addressed to Bing-Bing QIU, qiubb2005@163.com
}

Received 15 March 2012; Revised 3 June 2012; Accepted 4 July 2012

Academic Editor: Jun Hu

Copyright (C) 2012 W.-M. MA and B.-B. QIU. This is an open access article distributed under the Creative Commons Attribution License, which permits unrestricted use, distribution, and reproduction in any medium, provided the original work is properly cited.

Based on the mean and the standard deviation of lead time demand, and also taking the difficulty in measuring shortage cost into consideration, we investigate the joint decision problem of continuous review inventory in which a service level constraint should be satisfied. Under the assumption of controllable lead time and setup cost, a mathematical programming model is established. The objective function of the proposed model is the total expected annual cost and the constraint guarantees that the service level requirement can be satisfied at the worst case. Subsequently, an equivalent nonlinear programming model is derived. By constructing Lagrange function, the analysis regarding the solution procedure is conducted, and a solution algorithm is then provided. Moreover, a numerical example is introduced to illustrate the proposed model and solution algorithm. Through sensitivity analysis, some observations and managerial implications are provided.

\section{Introduction}

In inventory management, the length of lead time has direct influence on customer service level and total inventory cost. With the increasing competition in today's business environment, plenty of enterprises have devoted their efforts to pursuing a short lead time to enhance market competition ability. It is no doubt that the achievement of a shortened lead time requires a number of capital investments. Thus, some researchers have paid their attentions to balancing benefits and costs resulting from the reduction of lead time, and developed some theoretical models for possible decision aid. For example, Liao and Shyu [1] regarded the lead time as a decision variable. By assuming that the lead time composes of several components and the crashing cost is a linear function in the length of lead time concerning each component, a mathematical programming model with controllable lead time 
was constructed. Later, with the lead time crashing cost function proposed by Liao and Shyu [1], a lot of work has been done to develope some optimization models and algorithms in various decision environments for continuous inventory problems with variable lead time, such as Hariga and Ben-Daya [2], Ouyang and Chang [3], Wu and Ouyang [4], Yang et al. [5], Lee [6], Hoque and Goyal [7], and Annadurai and Uthayakumar [8]. However, the piecewise linear expression of lead time crashing cost has some deficiencies in application. Thus, BenDaya and Raouf [9] adopted negative exponential function to describe the lead time crashing cost and proposed a corresponding continuous review inventory model. Subsequently, Wu et al. [10] employed the negative exponential lead time crashing cost to develop a continuous review inventory model in which the lead time demand with the mixture of distributions was taken into account. Besides, Yang [11] proposed a supply chain integrated inventory model in the present of time value. In the proposed model, the lead time crashing cost was also assumed to be nonlinear in the length of lead time.

Likewise, in many real inventory problems, the setup cost could be reduced through increasing labor, improving facilities or adopting other relevant measures. In view of this point, Ouyang et al. [12] considered the partial backorder and proposed a modified continuous review inventory model with controllable lead time and setup cost. Taking the imperfect production process into account, Ouyang and Chang [13] constructed an inventory optimization model with controllable lead time and setup cost. In their research, both logarithmic and power investment functions were considered. With the assumption of controllable lead time and setup cost, Ouyang et al. [14] considered quality improvement in imperfect production process and investigated the associated inventory decision problem. Chuang et al. [15] assumed that the lead time demand is distribution-free in protection level and presented an inventory optimization model with variable lead time and setup cost. Taking the inconsistency between the receiving quantity and the ordering quantity into account, $\mathrm{Wu}$ and Lin [16] proposed an extended continuous review inventory model in which both lead time and ordering cost were variable. Subsequently, in supply chain setting, Chang et al. [17] proposed two integrated inventory models with the reductions of lead time and ordering cost. Considering the backorder discount, Lee et al. [18] developed a joint inventory decision model with variable lead time and ordering cost. In the research conducted by Uthayakumar and Parvathi [19], not only lead time and setup cost, but also yield variability was assumed to be variable. Besides, the backorder rate was assumed to be controllable through the amount of expected shortage. In their models, all the capital investments were assumed to be subject to logarithmic function. Annadurai and Uthayakumar [20] took the imperfect quality into account and developed a continuous review inventory model involving variable lead time and setup cost.

It is inevitable that shortage takes place with the assumption of stochastic lead time demand in continuous review inventory. However, in some practical situations, the shortage cost is difficult to estimate and therefore a service level constraint is announced by manager instead. Thus, based on different service level metrics, Aardal et al. [21] studied the optimal replenishment problem of continuous review inventory system. Moreover, some convex programming formulations were developed, and the associated solution algorithms were also given. With the normally distributed lead time demand, Ouyang and $\mathrm{Wu}$ [22] established a continuous review inventory model involving controllable lead time. In their research, a service level constraint was taken into account. Then, the assumption of normal distribution on lead time demand was relaxed and a distribution-free computational procedure was developed. By using the mixture of distributions to describe lead time demand, Lee et al. [23] proposed a continuous review inventory model with variable 
backorder rate and service level constraint. Jha and Shanker [24] proposed a model to solve ordering quantity, length of lead time and number of shipments in supply chain environment. In the concerned problem, controllable lead time and service level constraint were taken into consideration. Tajbakhsh [25] studied a distribution-free inventory model with a fill rate constraint. By solving the proposed model, the closed-form expressions of ordering quantity and reorder point were derived. Hsu and Huang [26] developed a distributionfree continuous review inventory model with multi-retailer. Moreover, both controllable lead time and service level constraint were considered. In Annadurai and Uthayakumar [27], and Jaggi and Arneja [28], with a service level constraint, the continuous review inventory models involving controllable lead time and setup cost were investigated. The former focused on the demand with the mixture of distributions, while the latter focused on the demand with normal distribution. More recently, Lin [29] presented a continuous review inventory model with a service level constraint. In the proposed model, setup cost, backorder rate, and lead time were assumed to be controllable. One of the same features in the models proposed by Annadurai and Uthayakumar [27], Jaggi and Arneja [28], and Lin [29] is that the piecewise linear lead time crashing cost was adopted. Besides, they derived safety coefficient from the allowable stock-out probability during lead time and thus the safety coefficient is not a decision variable. In fact, safety coefficient could be optimized, such as in Hariga and BenDaya [2], Wu and Ouyang [4], Hoque and Go [7], Annadurai and Uthayakumar [8, 20], Ouyang et al. [12, 14], Ouyang and Chang [3, 13], Chang et al. [17], Aardal et al. [21] and Tajbakhsh [25].

In this paper, we develop a continuous review inventory model with controllable lead time and setup cost. The lead-time-dependent cost is assumed to be a power function in the length of lead time, and the capital investment in setup cost reduction is assumed to follow a logarithmic expression. Moreover, the safety coefficient is treated as a decision variable. This disposition definitely leads to a more complex procedure of analyzing and deriving the optimal solution. In consideration of the difficulty in providing a precise estimation on the probability density function (p.d.f.) due to the insufficiency of historical data, we propose a distribution-free model according to the mean and the standard deviation of lead time demand. By constructing Lagrange function, we develop a solution procedure to determine ordering quantity, reorder point, length of lead time, and setup cost. Furthermore, we resolve a numerical example by using the proposed solution procedure and analyze the effects of the lower bound of service level.

The rest of this paper is organized as follows. In Section 2, we list the basic notations and assumptions used throughout this paper. In Section 3, the expression of total expected annual cost is firstly provided, and then a mathematical model of the concerned problem is proposed. In Section 4, an equivalent nonlinear programming formulation is derived. Moreover, a Lagrange function is constructed to obtain the optimal solution of the proposed model, and a solution algorithm is given. In Section 5, we resolve a numerical example by using the proposed solution algorithm. Through sensitivity analysis, some observations and managerial implications are presented. Finally, in Section 6, we summarize the whole paper and point out the next research work.

\section{Notations and Assumptions}

Before further development, we list the following notations which will be used throughout the paper. 
$q$ : ordering quantity, a decision variable.

$k$ : safety coefficient, $k \geq 0$, a decision variable.

$L$ : length of lead time, a decision variable.

$A$ : setup cost, a decision variable.

$X$ : lead time demand, a random variable.

$d$ : demand rate per year.

$f_{X}$ : probability density function of lead time demand.

$\sigma \sqrt{L}$ : standard derivation of lead time demand.

$r$ : reorder point.

$A_{0}$ : original setup cost.

SL: service level.

$\beta$ : lower bound of service level.

$R(L)$ : lead-time-dependent cost.

$I(A)$ : capital investment in setup cost reduction.

$\gamma$ : fractional opportunity cost per unit capital per year.

$h$ : holding cost per unit item per year.

EAC: total expected annual cost.

Moreover, the present problem is based on the following assumptions.

(1) Inventory is continuously monitored. Whenever the inventory level drops to a target value, an order is placed.

(2) The probability density function with regard to lead time demand is unknown.

(3) The service level is scaled by the fill rate which is defined as the fraction of demand satisfied from stock. Mathematically,

$$
S L=1-\frac{E(X-r)^{+}}{q} .
$$

in which $E(\cdot)$ is the mathematical expectation and $x^{+}=\max (x, 0)$.

(4) The reorder point is determined by $r=d L+k \sigma \sqrt{L}$, in which $k \sigma \sqrt{L}$ denotes safety inventory.

(5) The lead-time-dependent cost follows a power function. Mathematically,

$$
R(L)=a L^{-b},
$$

in which $a>0$ and $b>0$ are constants. 
(6) The capital investment in setup cost reduction follows a logarithmic function. Mathematically,

$$
I(A)=\frac{1}{\delta} \ln \left(\frac{A_{0}}{A}\right), \quad 0<A \leq A_{0}
$$

in which $\delta>0$ is a constant.

(7) As in Tajbakhsh [25], we assume that the lower bound of service level satisfies $1 / 2<\beta<1$. This value range is quite reasonable in application.

\section{The Mathematical Model}

Herein, we intend to provide a feasible solution scheme for the joint decision problem in continuous review inventory with a service level constraint. For the present problem, it is assumed that both lead time and setup cost can be reduced through capital expenditures. Hence, the decision variables contain not only ordering quantity and safety coefficient, but also length of lead time and setup cost.

Based on the previous description, the length of cycle is $q / d$, and the setup cost per cycle is $A$. In continuous review inventory system, an order with size $q$ is placed when the inventory level drops to the reorder point $r$, and the order is received at the end of lead time. Thus, the inventory holding cost per year is $(h) /(2)[q+2(r-d L)]$, in which $d L$ denotes the mean of lead time demand.

Taking the capital expenditures related to the reductions of lead time and setup cost into account, we can formulate the expression of total expected annual cost as follows:

$$
\operatorname{EAC}(q, r, A, L)=\frac{d}{q}\left(A+a L^{-b}\right)+\frac{h}{2}[q+2(r-d L)]+\frac{\gamma}{\delta} \ln \left(\frac{A_{0}}{A}\right)
$$

Generally speaking, the precise estimation of probability density function on lead time demand requires enough adequate data, which is difficult to realize in application. Therefore, in the development of the proposed model, we do not make any assumptions on the distribution function of lead time demand. Namely, we focused on the case when the specific distribution of lead time demand is unavailable.

Denote the collection of probability density function $f_{X}$ with the mean $d L$ and the standard derivation $\sigma \sqrt{L}$ by $F$. Assuming the manager is conservative and expects that the service level constraint holds for all possible probability distributions, we get

$$
\underset{f_{X} \in F}{\operatorname{minimize}}\left\{1-\frac{E(X-r)^{+}}{q}\right\} \geq \beta
$$

The above disposition to service level constraint is in line with Tajbakhsh [25] and reflects robustness. Similar philosophy was widely adopted in the control of complex systems, such as Bausoa et al. [30], Dong et al. [31], Jaśkiewicz and Nowak [32] and 
Hu et al. [33]. Furthermore, taking the total expected annual cost as objective function, we can establish the following programming model for the present problem:

$$
\begin{array}{cl}
\underset{q, r, A, L}{\operatorname{minimize}} & \operatorname{EAC}(q, r, A, L) \\
\text { subject to } \underset{f_{X} \in F}{\operatorname{maximize}} & E(X-r)^{+} \leq(1-\beta) q \\
& q \geq 0, \quad r \geq d L, \quad 0<A \leq A_{0}, \quad L>0 .
\end{array}
$$

\section{Solution Procedure}

To facilitate further exploration, we introduce the following proposition to eliminate the max operator in the service level constraint of model (3.3).

Proposition 4.1. Given the mean $d L$ and the standard derivation $\sigma \sqrt{L}$ of lead time demand $X$, then

$$
E(X-r)^{+} \leq \frac{\sigma \sqrt{L}\left(\sqrt{k^{2}+1}-k\right)}{2} .
$$

Moreover, there is at least a p.d.f. which makes the equal sign in (4.1) holds.

Proposition 4.1 is similar to Lemma 1 in Gallego and Moon [34]. Therefore, we omit the proof procedure. In light of Proposition 4.1, and substituting the relation $r=d L+k \sigma \sqrt{L}$ into the objective function of model (3.3), we get an equivalent nonlinear programming model:

$$
\begin{aligned}
& \underset{q, k, A, L}{\operatorname{minimize}} \operatorname{EAC}(q, k, A, L)=\frac{d}{q}\left(A+a L^{-b}\right)+\frac{h}{2}(q+2 k \sigma \sqrt{L})+\frac{\gamma}{\delta} \ln \left(\frac{A_{0}}{A}\right) \\
& \text { subject to } \sigma \sqrt{L}\left(\sqrt{k^{2}+1}-k\right)-2(1-\beta) q \leq 0 \\
& \qquad q \geq 0, \quad k \geq 0, \quad 0<A \leq A_{0}, \quad L>0 .
\end{aligned}
$$

To solve model (4.2), a Lagrange function is constructed as follows:

$$
\begin{aligned}
F(q, k, A, L, \lambda)= & \frac{d}{q}\left(A+a L^{-b}\right)+\frac{h}{2}(q+2 k \sigma \sqrt{L})+\frac{\gamma}{\delta} \ln \left(\frac{A_{0}}{A}\right) \\
& +\lambda\left[\sigma \sqrt{L}\left(\sqrt{k^{2}+1}-k\right)-2(1-\beta) q\right],
\end{aligned}
$$

in which $\lambda \geq 0$ is a Lagrange multiplier.

Then, with the first order optimality condition $\partial F(q, k, A, L, \lambda) / \partial k=0$, we get

$$
\lambda=\frac{h \sqrt{k^{2}+1}}{\sqrt{k^{2}+1}-k} .
$$


From (4.4), we conclude that the Lagrange multiplier should satisfy $\lambda \geq h$. This relationship manifests that the service level constraint is never inactive. Thus, the optimal solution should satisfy the following equation:

$$
\sigma \sqrt{L}\left(\sqrt{k^{2}+1}-k\right)-2(1-\beta) q=0
$$

Equivalently,

$$
q=\frac{\sigma \sqrt{L}\left(\sqrt{k^{2}+1}-k\right)}{2(1-\beta)}
$$

Let $\partial F(q, k, A, L, \lambda) / \partial L=0$ and yield:

$$
2 a b d-\sigma q L^{b+1 / 2}\left[h k+\lambda\left(\sqrt{k^{2}+1}-k\right)\right]=0 .
$$

Substituting (4.4) and (4.6) into (4.7), after some algebraic manipulation, we get

$$
L_{1 *}=\left[\frac{4 a b d(1-\beta)}{h \sigma^{2}}\right]^{1 /(b+1)}
$$

Furthermore, by letting $\partial F(q, k, A, L, \lambda) /(\partial A)=0$, we obtain

$$
q=\frac{\delta d A}{r}
$$

Combining (4.4), (4.6), and (4.9), we have

$$
\lambda=\frac{h L \sigma^{2} \gamma^{2}}{8 \delta^{2} d^{2} A^{2}(1-\beta)^{2}}+\frac{h}{2}
$$

Then, let $\partial F(q, k, A, L, \lambda) / \partial q=0$ and get

$$
2 d\left(A+a L^{-b}\right)+q^{2}[4(1-\beta) \lambda-h]=0 .
$$

Then, with the length of lead time from (4.8), and substituting (4.9) and (4.10) into (4.11), we get the following quadratic equation with respect to setup cost $A$ :

$$
A^{2}-\frac{2 \gamma^{2} A}{d h \delta^{2}(2 \beta-1)}-\frac{\gamma^{2}\left[h \sigma^{2} L_{1 *}^{b+1}+4 a d(1-\beta)\right]}{2 h \delta^{2} d^{2} L_{1 *}^{b}(1-\beta)(2 \beta-1)}=0 .
$$


Again, with the assumption $1 / 2<\beta<1$, it is obvious that the above equation has a unique positive root. Due to the complex formulation of (4.12), we do not write the analytical expression of its solution. However, with the given parameters, it is easy to resolve (4.12) and get the value of setup cost. For the convenience of description in the sequel, we denote the unique positive solution of (4.12) by $A_{1 *}$.

With the resultant setup cost $A_{1 *}$ and the length of lead time $L_{1 *}$, we can determine the corresponding ordering quantity $q_{1 *}$ and the Lagrange multiplier $\lambda_{1 *}$,respectively, by using (4.9) and (4.10). Namely, $q_{1 *}=\delta d A_{1 *} / \gamma$ and $\lambda_{1 *}=h L \sigma^{2} \gamma^{2} / 8 \delta^{2} d^{2} A^{2}{ }_{1 *}(1-\beta)^{2}+h / 2$.

Subsequently, the safety coefficient can be obtained by the following formula:

$$
k_{1 *}=\frac{\lambda_{1 *}-h}{\sqrt{h\left(2 \lambda_{1 *}-h\right)}} .
$$

Then, we need to examine whether the solution $\left(q_{1 *}, k_{1 *}, A_{1 *}, L_{1 *}\right)$ is a minimum. Although it is difficult to verify that the nonlinear programming model (4.2) is convex, we can demonstrate that the Hessian of Lagrangian is positive definition at point $\left(q_{1 *}, k_{1 *}, A_{1 *}, L_{1 *}, \lambda_{1 *}\right)$. To this end, the Hessian matrix is written as follows:

$$
H=\left[\begin{array}{llll}
\frac{\partial^{2} F(q, k, A, L, \lambda)}{\partial q^{2}} & \frac{\partial^{2} F(q, k, A, L, \lambda)}{\partial q \partial k} & \frac{\partial^{2} F(q, k, A, L, \lambda)}{\partial q \partial A} & \frac{\partial^{2} F(q, k, A, L, \lambda)}{\partial q \partial L} \\
\frac{\partial^{2} F(q, k, A, L, \lambda)}{\partial k \partial q} & \frac{\partial^{2} F(q, k, A, L, \lambda)}{\partial k^{2}} & \frac{\partial^{2} F(q, k, A, L, \lambda)}{\partial k \partial A} & \frac{\partial^{2} F(q, k, A, L, \lambda)}{\partial k \partial L} \\
\frac{\partial^{2} F(q, k, A, L, \lambda)}{\partial A \partial q} & \frac{\partial^{2} F(q, k, A, L, \lambda)}{\partial A \partial k} & \frac{\partial^{2} F(q, k, A, L, \lambda)}{\partial A^{2}} & \frac{\partial^{2} F(q, k, A, L, \lambda)}{\partial A \partial L} \\
\frac{\partial^{2} F(q, k, A, L, \lambda)}{\partial L \partial q} & \frac{\partial^{2} F(q, k, A, L, \lambda)}{\partial L \partial k} & \frac{\partial^{2} F(q, k, A, L, \lambda)}{\partial L \partial A} & \frac{\partial^{2} F(q, k, A, L, \lambda)}{\partial L^{2}}
\end{array}\right] .
$$

Moreover,

$$
\begin{gathered}
\frac{\partial^{2} F\left(q_{1 *}, k_{1 *}, A_{1 *}, L_{1 *}, \lambda_{1 *}\right)}{\partial q^{2}}=\frac{2 d\left(a+A_{1 *} L_{1 *}^{b}\right)}{q_{1 *}^{3} L_{1 *}^{b}}, \\
\frac{\partial^{2} F\left(q_{1 *}, k_{1 *}, A_{1 *}, L_{1 *}, \lambda_{1 *}\right)}{\partial k^{2}}=\frac{\lambda \sigma \sqrt{L_{1 *}}}{\left(k_{1 *}^{2}+1\right)^{3 / 2}}, \\
\frac{\partial^{2} F\left(q_{1 *}, k_{1 *}, A_{1 *}, L_{1 *}, \lambda_{1 *}\right)}{\partial A^{2}}=\frac{\gamma}{\delta A_{1 *}^{2}} \\
=\frac{d}{q_{1 *} A_{1 *}}, \\
\frac{\partial^{2} F\left(q_{1 *}, k_{1 *}, A_{1 *}, L_{1 *}, \lambda_{1 *}\right)}{\partial L^{2}}=\frac{a b d(b+1)}{q_{1 *} L_{1 *}^{b+2}}+\frac{\sigma\left[h k_{1 *}+\lambda\left(\sqrt{k_{1 *}^{2}+1}-k_{1 *}\right)\right]}{4 L_{1 *}^{3 / 2}} \\
=\frac{a b d(2 b+3)}{2 q_{1 *} L_{1 *}^{b+2}},
\end{gathered}
$$


Mathematical Problems in Engineering

$$
\begin{aligned}
& \frac{\partial^{2} F\left(q_{1 *}, k_{1 *}, A_{1 *}, L_{1 *}, \lambda_{1 *}\right)}{\partial q \partial k}=\frac{\partial^{2} F\left(q_{1 *}, k_{1 *}, A_{1 *}, L_{1 *}, \lambda_{1 *}\right)}{\partial k \partial q} \\
& =\frac{\partial^{2} F\left(q_{1 *}, k_{1 *}, A_{1 *}, L_{1 *}, \lambda_{1 *}\right)}{\partial k \partial A} \\
& =\frac{\partial^{2} F\left(q_{1 *}, k_{1 *}, A_{1 *}, L_{1 *}, \lambda_{1 *}\right)}{\partial A \partial k} \\
& =\frac{\partial^{2} F\left(q_{1 *}, k_{1 *}, A_{1 *}, L_{1 *}, \lambda_{1 *}\right)}{\partial A \partial L} \\
& =\frac{\partial^{2} F\left(q_{1 *}, k_{1 *}, A_{1 *}, L_{1 *}, \lambda_{1 *}\right)}{\partial L \partial A} \\
& =0 \text {, } \\
& \frac{\partial^{2} F\left(q_{1 *}, k_{1 *}, A_{1 *}, L_{1 *}, \lambda_{1 *}\right)}{\partial q \partial A}=\frac{\partial^{2} F\left(q_{1 *}, k_{1 *}, A_{1 *}, L_{1 *}, \lambda_{1 *}\right)}{\partial A \partial q} \\
& =-\frac{d}{q_{1 *}^{2}}, \\
& \frac{\partial^{2} F\left(q_{1 *}, k_{1 *}, A_{1 *}, L_{1 *}, \lambda_{1 *}\right)}{\partial q \partial L}=\frac{\partial^{2} F\left(q_{1 *}, k_{1 *}, A_{1 *}, L_{1 *}, \lambda_{1 *}\right)}{\partial L \partial q} \\
& =\frac{a b d}{q_{1 *}^{2} L_{1 *}^{b+1}}, \\
& \frac{\partial^{2} F\left(q_{1 *}, k_{1 *}, A_{1 *}, L_{1 *}, \lambda_{1 *}\right)}{\partial k \partial L}=\frac{\partial^{2} F\left(q_{1 *}, k_{1 *}, A_{1 *}, L_{1 *}, \lambda_{1 *}\right)}{\partial L \partial k} \\
& =\frac{\sigma\left[\left(h-\lambda_{1 *}\right) \sqrt{k_{1 *}^{2}+1}+\lambda_{1 *} k_{1 *}\right]}{2 \sqrt{L_{1 *}\left(k_{1 *}^{2}+1\right)}} \\
& =0 \text {. }
\end{aligned}
$$

It is worth mentioning that the second equal sign in (4.17) is based on (4.9), the second equal sign in (4.18) is based on (4.4), (4.6) and (4.8), and the third equal sign in (4.22) is based on (4.4).

In light of (4.15)-(4.22), the first and second principal minor determinants of matrix $H$ at point $\left(q_{1 *}, k_{1 *}, A_{1 *}, L_{1 *}, \lambda_{1 *}\right)$ are obviously positive, which are shown as follows:

$$
\begin{aligned}
\left|H_{11}\right| & =\frac{2 d\left(a+A_{1 *} L_{1 *}^{b}\right)}{q_{1 *}^{3} L_{1 *}^{b}} \\
& \geq 0,
\end{aligned}
$$




$$
\begin{aligned}
\left|H_{22}\right| & =\frac{2 \lambda \sigma d\left(a+A_{1 *} L_{1 *}^{b}\right)}{q_{1 *}^{3} L_{1 *}^{b-1 / 2}\left(k_{1 *}^{2}+1\right)^{3 / 2}} \\
& \geq 0 .
\end{aligned}
$$

And the third principal minor determinant of $H$ at point $\left(q_{1 *}, k_{1 *}, A_{1 *}, L_{1 *}, \lambda_{1 *}\right)$ is computed as follows:

$$
\begin{aligned}
\left|H_{33}\right| & =\frac{2 \lambda \sigma d^{2}\left(a+A_{1 *} L_{1 *}^{b}\right)}{A_{1 *} q_{1 *}^{4} L_{1 *}^{b-1 / 2}\left(k_{1 *}^{2}+1\right)^{3 / 2}}-\frac{\lambda \sigma d^{2} \sqrt{L_{1 *}}}{q_{1 *}^{4}\left(k_{1 *}^{2}+1\right)^{3 / 2}} \\
& =\frac{\lambda \sigma d^{2}\left(2 a+A_{1 *} L_{1 *}^{b}\right)}{A_{1 *} q_{1 *}^{4} L_{1 *}^{b-1 / 2}\left(k_{1 *}^{2}+1\right)^{3 / 2}} \\
& \geq 0 .
\end{aligned}
$$

In addition, the fourth principal minor determinant of Hessian matrix $H$ at point $\left(q_{1 *}, k_{1 *}, A_{1 *}, L_{1 *}, \lambda_{1 *}\right)$ is computed as follows:

$$
\begin{aligned}
\left|H_{44}\right| & =\frac{\lambda \sigma a b d^{3}(2 b+3)\left(a+A_{1 *} L_{1 *}^{b}\right)}{A_{1 *} q_{1 *}^{5} L_{1 *}^{2 b+3 / 2}\left(k_{1 *}^{2}+1\right)^{3 / 2}}-\frac{\lambda \sigma a b d^{3}(2 b+3)}{2 q_{1 *}^{5} L_{1 *}^{b+3 / 2}\left(k_{1 *}^{2}+1\right)^{3 / 2}} \\
& =\frac{\lambda \sigma a b d^{3}(2 b+3)\left(2 a+A_{1 *} L_{1 *}^{b}\right)}{2 A_{1 *} q_{1 *}^{5} L_{1 *}^{2 b+3 / 2}\left(k_{1 *}^{2}+1\right)^{3 / 2}} \\
& \geq 0 .
\end{aligned}
$$

Therefore, according to the second order sufficient conditions (SOSCs) [35], $\left(q_{1 *}, k_{1 *}, A_{1 *}, L_{1 *}\right)$ is a minimum.

Notice that the interval and nonnegative constraints of model (4.2) are ignored while constructing Lagrange function (4.3). Thus, if the setup cost derived using (4.12) does not make the inequality $A_{1 *} \leq A_{0}$ hold, we need to take the following special case into account, that is, $A_{2 *}=A_{0}$.

For the case of $A_{2 *}=A_{0}$, it means that, to minimize the total expected annual cost, the manager need not adopt any actions to reduce the setup cost. Thus, similar to the above 
deduction procedure, we can achieve the length of lead time and the ordering quantity through the following equations:

$$
\begin{gathered}
L_{2 *}=\left[\frac{4 a b d(1-\beta)}{h \sigma^{2}}\right]^{1 /(b+1)}, \\
q_{2 *}=\sqrt{\frac{4 d(1-\beta)\left(a+A_{2 *} L_{2 *}^{b}\right)+h \sigma^{2} L_{2 *}^{b+1}}{2(1-\beta)(2 \beta-1) h L_{2 *}^{b}}} .
\end{gathered}
$$

Then, the Lagrange multiplier is

$$
\lambda_{2 *}=\frac{h}{4(1-\beta)}-\frac{d\left(a+A_{2 *} L_{2 *}^{b}\right)}{2(1-\beta) q_{2 *}^{2} L_{2 *}^{b}} .
$$

Accordingly, the safety coefficient is

$$
k_{2 *}=\frac{\lambda_{2 *}-h}{\sqrt{h\left(2 \lambda_{2 *}-h\right)}} .
$$

In accordance with Aardal et al. [21], we confine the present discussion to the service level which yields $k>0$. Actually, with a similar computational procedure, it is easy to determine the optimal values of ordering quantity, length of lead time, and setup cost for the case of $k=0$. To keep compact, we do not provide the details.

According to the above analysis procedure, the solution algorithm for the proposed model is summarized as follows.

Step 1. Determine the values of $L_{1 *}$ and $A_{1 *}$, respectively, by using (4.8) and (4.12).

Step 2. If $A_{1 *}>A_{0}$, go to Step 3. Otherwise, determine the values of $q_{1 *}, \lambda_{1 *}$, and $k_{1 *}$, respectively, by using (4.9), (4.10), and (4.13). Let $q^{*}=q_{1 *}, k^{*}=k_{1 *}, A^{*}=A_{1 *}, L^{*}=L_{1 *}$. Go to Step 4.

Step 3. Set $A_{2 *}=A_{0}$ and determine the values of $L_{2 *}$ and $q_{2 *}$ by using (4.27) and (4.28). Then, determine the values of $\lambda_{2 *}$ and $k_{2 *}$, respectively, by using (4.28) and (4.29). Let $q^{*}=q_{2 *}$, $k^{*}=k_{2 *}, A^{*}=A_{2 *}, L^{*}=L_{2 *}$.

Step 4. End.

\section{Numerical Example}

In this section, a numerical example is utilized to demonstrate the feasibility of the proposed solution procedure. Moreover, we will vary the lower bound of service level to perform 


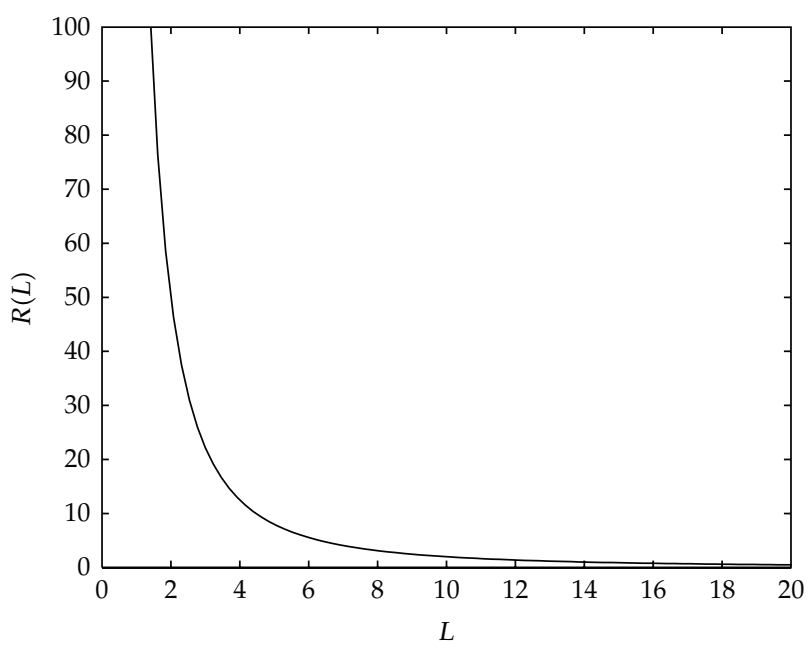

Figure 1: The lead-time-dependent cost.

sensitivity analysis and give some observations and managerial implications. The basic parameters are as follows:

$$
\begin{aligned}
& \beta=0.975, \\
& \gamma=0.1 / \text { dollar/year, } \\
& d=700 \text { units/year, } \\
& \sigma=15 \text { units/week, } \\
& h=\$ 25 / \text { year/unit, } \\
& A_{0}=\$ 300 / \text { order. }
\end{aligned}
$$

The function expression of lead-time-dependent cost is $R(L)=1000 L^{-3}$ with $a=1000$ and $b=3$. Moreover, the function expression of capital investment in setup cost reduction is $I(A)=10000 \ln (300 / A)$ with $\delta=0.0001$. The curves of two capital investment functions are, respectively, depicted in Figures 1 and 2.

With the aforementioned data and function expressions, and using the proposed method, we can calculate the ordering quantity $q^{*}=115.59$ units, the safety coefficient $k^{*}=0.7293$, the length of lead time $L^{*}=28.14$ days and the setup cost $A^{*}=165.13$ dollars. Thus, the reorder point $r^{*}=62.27$ units, the lead-time-dependent cost $R^{*}(L)=15.39$ dollars, the capital investment in setup cost reduction $I^{*}(A)=5970.3$ dollars, and the total expected annual cost $\mathrm{EAC}^{*}=3342.4$ dollars.

Next, we vary the value of $\beta$ from 0.96 to 0.99 with equal interval 0.1 to perform sensitivity analysis. The computational results are shown in Table 1.

From the data in Table 1, several observations and managerial implications are made as follows:

(1) When the value of $\beta$ is varied from 0.96 to 0.99 , the ordering quantity and the safety coefficient increase while the reorder point decreases. Moreover, compared with the ordering quantity and the reorder point, the change in the safety coefficient is great. This phenomenon implies that the change in lower bound of service level has 


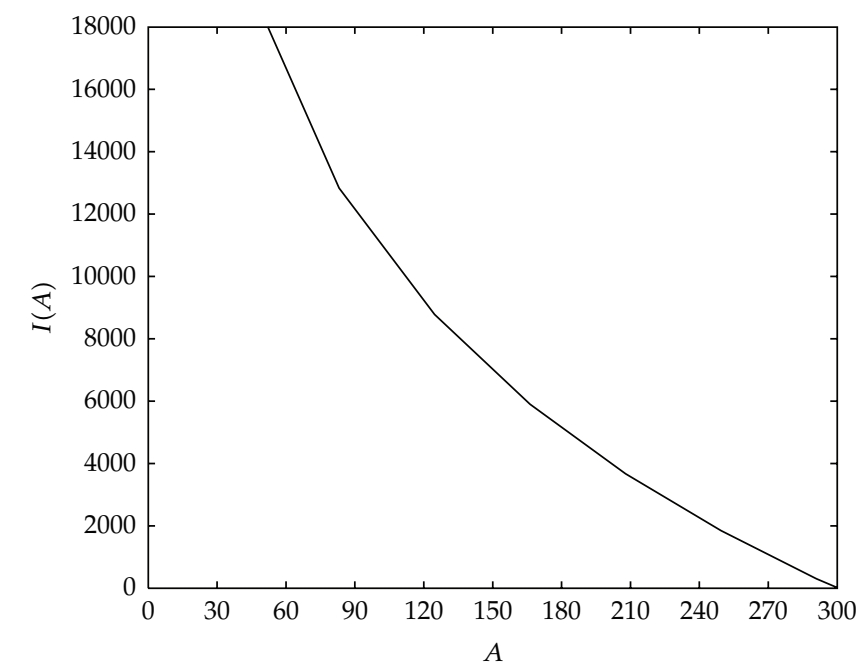

Figure 2: The capital investment function in setup cost reduction.

Table 1: Effect of change in parameter $\beta$.

\begin{tabular}{lcccc}
\hline$\beta$ & 0.96 & 0.97 & 0.98 & 0.99 \\
\hline$q^{*}$ & 110.74 & 113.32 & 119.00 & 133.86 \\
$k^{*}$ & 0.3131 & 0.5629 & 0.9460 & 1.7613 \\
$r^{*}$ & 64.48 & 63.04 & 61.51 & 60.78 \\
$L^{*}$ & 31.65 & 29.46 & 26.62 & 22.38 \\
$A^{*}$ & 158.19 & 161.89 & 170.00 & 191.23 \\
$R^{*}(L)$ & 10.81 & 13.42 & 18.19 & 30.59 \\
$I^{*}(A)$ & 6399.7 & 6168.6 & 5680.1 & 4502.9 \\
EAC $^{*}$ & 3186.9 & 3280.0 & 3423.9 & 3729.9 \\
\hline
\end{tabular}

a greater impact on the safety coefficient and the reorder point than the ordering quantity.

(2) We observe that a larger value of $\beta$ yields a shorter lead time, a higher lead-timedependent cost. This phenomenon indicates that the short lead time is favorable to the service level. Moreover, we also observe that a larger value of $\beta$ leads to a higher setup cost and a smaller value of capital investment in setup cost reduction.

(3) As the value of $\beta$ increases, the total expected annual cost also increases. It seems that a lower service level benefits manager in profit. However, the lower service will produce negative influences on brand and customer loyalty which are crucial to building competitive advantage in market. From this perspective, the manager should determine a proper lower bound of service level which can balance shortterm income and long-term development.

\section{Conclusions and Future Work}

Considering the difficulty in measuring shortage cost, we proposed a distribution-free continuous review inventory model in the presence of a service level constraint. In our model, 
the lead-time-dependent cost is assumed to be a power function in the length of lead time, and the capital investment in setup cost reduction is assumed to be a logarithmic function in setup cost. The proposed model guarantees that the service level constraint can be satisfied at the worst case and takes ordering quantity, safety coefficient, length of lead time and setup cost as decision variables. In the present research, we also discuss the optimal solution of the proposed model and develop an effective solution procedure. Moreover, the results contained in this research are illustrated and verified by a numerical example.

In the future research, we may take other forms of investment function into consideration. Besides, we will conduct some relevant research to extend the present model from many perspectives, such as imperfect quality, uncertain yield and so on, to develop some novel models and design the corresponding solution algorithms. Another feasible extension of the present research is to develop the inventory model and the associated solution algorithm by considering the interacted effect of capital investment in the reductions of lead time and setup cost. Additionally, in the research regarding continuous review inventory problems, the safety coefficient is usually assumed to be nonnegative. However, for some replenishment problems with short lead time and large ordering batch caused by high setup cost and low inventory holding cost, it may be economic to set a negative safety coefficient. For the negative safety coefficient, a different expression of inventory holding cost should be adopted, such as in Klouja and Antonis [36]. Therefore, it is also meaningful to relax the nonnegative assumption in safety coefficient and perform some extension on the results contained in this research.

\section{Acknowledgments}

The authors would like to express their sincere thanks to the editor and the anonymous reviewers for their valuable comments to this paper. This work was partially support by the National Natural Science Foundation of China (71071113), the Ph.D. Programs Foundation of Higher Education of China (20100072110011), the Foundation for the Author of National Excellent Doctoral Dissertation of PR China (200782), the Shanghai Pujiang Program, the Shanghai Philosophical and Social Science Program (2010BZH003), and the Fundamental Research Funds for the Central Universities.

\section{References}

[1] C. J. Liao and C. H. Shyu, "An analytical determination of lead time with normal demand," International Journal of Operations and Production Management, vol. 11, no. 9, pp. 72-78, 1991.

[2] M. Hariga and M. Ben-Daya, "Some stochastic inventory models with deterministic variable lead time," European Journal of Operational Research, vol. 113, no. 1, pp. 42-51, 1999.

[3] L. Y. Ouyang and B. R. Chuang, "Mixture inventory model involving variable lead time and controllable backorder rate," Computers and Industrial Engineering, vol. 40, no. 4, pp. 339-348, 2001.

[4] K. S. Wu and L. Y. Ouyang, "(Q,r,L) Inventory model with defective items," Computers and Industrial Engineering, vol. 39, no. 1-2, pp. 173-185, 2001.

[5] G. Yang, R. J. Ronald, and P. Chu, "Inventory models with variable lead time and present value," European Journal of Operational Research, vol. 164, no. 2, pp. 358-366, 2005.

[6] W.-C. Lee, "Inventory model involving controllable backorder rate and variable lead time demand with the mixtures of distribution," Applied Mathematics and Computation, vol. 160, no. 3, pp. 701-717, 2005.

[7] M. A. Hoque and S. K. Goyal, “An alternative simple solution algorithm of an inventory model with fixed and variable lead time crash costs under unknown demand distribution," International Journal of Systems Science, vol. 40, no. 8, pp. 821-827, 2009. 
[8] K. Annadurai and R. Uthayakumar, "Reducing lost-sales rate in $(T, R, L)$ inventory model with controllable lead time," Applied Mathematical Modelling, vol. 34, no. 11, pp. 3465-3477, 2010.

[9] M. Ben-Daya and A. Raouf, "Inventory models involving lead time as a decision variable," Journal of the Operational Research Society, vol. 45, no. 5, pp. 579-582, 1994.

[10] J.-W. Wu, W.-C. Lee, and H.-Y. Tsai, “Computational algorithmic procedure of optimal inventory policy involving a negative exponential crashing cost and variable lead time demand," Applied Mathematics and Computation, vol. 184, no. 2, pp. 798-808, 2007.

[11] M.-F. Yang, "Supply chain integrated inventory model with present value and dependent crashing cost is polynomial," Mathematical and Computer Modelling, vol. 51, no. 5-6, pp. 802-809, 2010.

[12] L. Y. Ouyang, C. K. Chen, and H. C. Chang, "Lead time and ordering cost reductions in continuous review inventory systems with partial backorders," Journal of the Operational Research Society, vol. 50, no. 12 , pp. $1272-1279,1999$.

[13] L.-Y. Ouyang and H.-C. Chang, "Lot size reorder point inventory model with controllable lead time and set-up cost," International Journal of Systems Science, vol. 33, no. 8, pp. 635-642, 2002.

[14] L. Y. Ouyang, C. K. Chen, and H. C. Chang, "Quality improvement, setup cost and lead-time reductions in lot size reorder point models with an imperfect production process," Computers and Operations Research, vol. 29, no. 12, pp. 1701-1717, 2002.

[15] B. R. Chuang, L. Y. Ouyang, and K. W. Chuang, "A note on periodic review inventory model with controllable setup cost and lead time," Computers and Operations Research, vol. 31, no. 4, pp. 549-561, 2004.

[16] K. S. Wu and I. C. Lin, "Extend $(r, Q)$ inventory model under lead time and ordering cost reductions when the receiving quantity is different from the ordered quantity," Quality \& Quantity, vol. 38, no. 6, pp. 771-786, 2004.

[17] H.-C. Chang, L.-Y. Ouyang, K.-S. Wu, and C.-H. Ho, "Integrated vendor-buyer cooperative inventory models with controllable lead time and ordering cost reduction," European Journal of Operational Research, vol. 170, no. 2, pp. 481-495, 2006.

[18] W.-C. Lee, J.-W. Wu, and C.-L. Lei, “Computational algorithmic procedure for optimal inventory policy involving ordering cost reduction and back-order discounts when lead time demand is controllable," Applied Mathematics and Computation, vol. 189, no. 1, pp. 186-200, 2007.

[19] R. Uthayakumar and P. Parvathi, "A continuous review inventory model with controllable backorder rate and investments," International Journal of Systems Science, vol. 40, no. 3, pp. 245-254, 2009.

[20] K. Annadurai and R. Uthayakumar, "Controlling setup cost in $(Q, r, L)$ inventory model with defective items," Applied Mathematical Modelling, vol. 34, no. 6, pp. 1418-1427, 2010.

[21] K. Aardal, O. Jonsson, and H. Jonsson, "Optimal inventory policies with service-level constraints," The Journal of the Operational Research Society, vol. 40, no. 1, pp. 65-73, 1989.

[22] L.-Y. Ouyang and K.-S. Wu, "Mixture inventory model involving variable lead time with a service level constraint," Computers E Operations Research, vol. 24, no. 9, pp. 875-882, 1997.

[23] W.-C. Lee, J.-W. Wu, and J.-W. Hsu, "Computational algorithm for inventory model with a service level constraint, lead time demand with the mixture of distributions and controllable negative exponential backorder rate," Applied Mathematics and Computation, vol. 175, no. 2, pp. 1125-1138, 2006.

[24] J. K. Jha and K. Shanker, "Two-echelon supply chain inventory model with controllable lead time and service level constraint," Computers and Industrial Engineering, vol. 57, no. 3, pp. 1096-1104, 2009.

[25] M. M. Tajbakhsh, "On the distribution free continuous-review inventory model with a service level constraint," Computers and Industrial Engineering, vol. 59, no. 4, pp. 1022-1024, 2010.

[26] S.-L. Hsu and Y.-F. Huang, "An integrated inventory model with controllable lead time and distribution-free demand," Applied Stochastic Models in Business and Industry, vol. 26, no. 4, pp. 416$430,2010$.

[27] K. Annadurai and R. Uthayakumar, "Ordering cost reduction in probabilistic inventory model with controllable lead time and a service level," International Journal of Management Science and Engineering Management, vol. 5, no. 6, pp. 403-410, 2010.

[28] C. K. Jaggi and N. Arneja, "Periodic inventory model with reduced setup cost under service level constraint," Electronic Journal of Applied Statistical Analysis, vol. 4, no. 2, pp. 111-123, 2011.

[29] H. J. Lin, "Effective investment to reduce setup cost in a mixture inventory model involving controllable backorder rate and variable lead time with a service level constraint," Mathematical Problems in Engineering, vol. 2012, Article ID 689061, 15 pages, 2012.

[30] D. Bauso, F. Blanchini, and R. Pesenti, "Robust control strategies for multi-inventory systems with average flow constraints," Automatica, vol. 42, no. 8, pp. 1255-1266, 2006. 
[31] H. Dong, Z. Wang, D. W. C. Ho, and H. Gao, "Robust Ho fuzzy output-feedback control with multiple probabilistic delays and multiple missing measurements," IEEE Transactions on Fuzzy Systems, vol. 18, no. 4, pp. 712-725, 2010.

[32] A. Jaśkiewicz and A. S. Nowak, "Stochastic games with unbounded payoffs: applications to robust control in economics," Dynamic Games and Applications, vol. 1, no. 2, pp. 253-279, 2011.

[33] J. Hu, Z. Wang, H. Gao, and L. K. Stergioulas, "Robust sliding mode control for discrete stochastic systems with mixed time delays, randomly occurring uncertainties, and randomly occurring nonlinearities," IEEE Transactions on Industrial Electronics, vol. 59, no. 7, pp. 3008-3015, 2012.

[34] G. Gallego and I. Moon, "Distribution free newsboy problem: review and extensions," Journal of the Operational Research Society, vol. 44, no. 8, pp. 825-834, 1993.

[35] D. P. Bertsekas, Nonlinear Programming, Athena Scientific, Belmont, Mass, USA, 1995.

[36] M. Khouja and A. C. Stylianou, "A ( $Q, R)$ inventory model with a drop-shipping option for ebusiness," Omega, vol. 37, no. 4, pp. 896-908, 2009. 


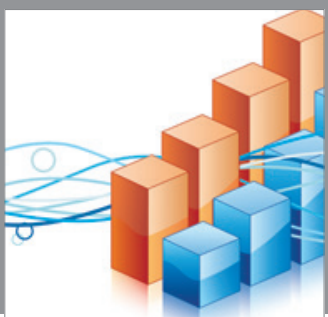

Advances in

Operations Research

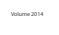

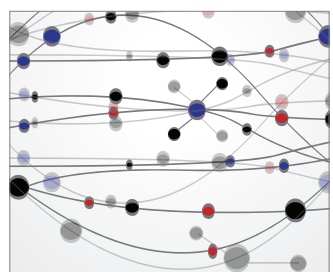

\section{The Scientific} World Journal
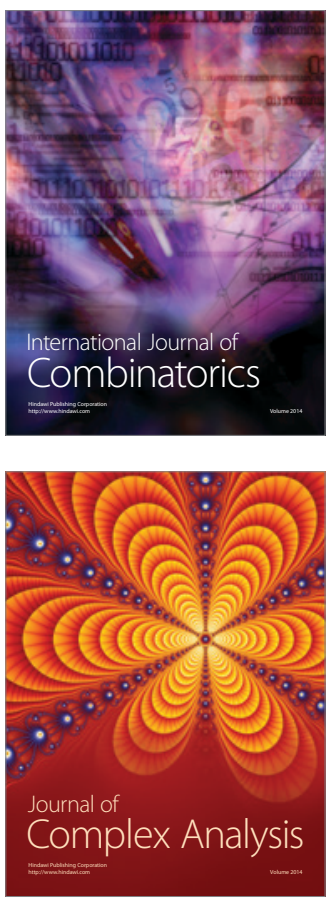

International Journal of

Mathematics and

Mathematical

Sciences
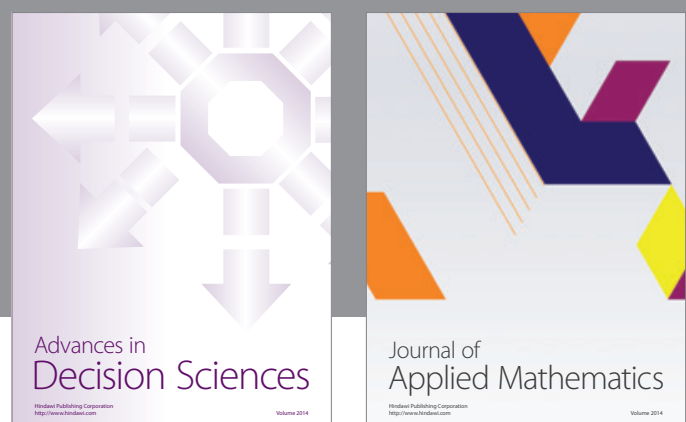

Journal of

Applied Mathematics
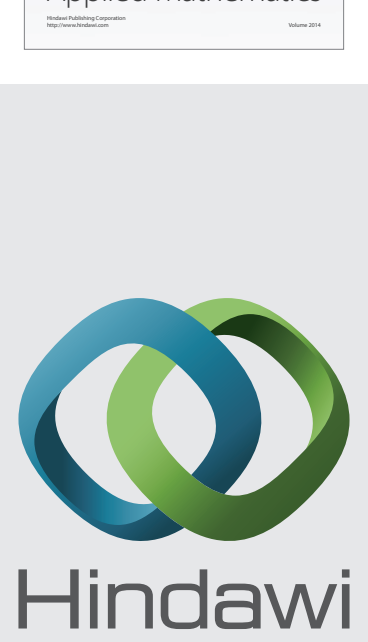

Submit your manuscripts at http://www.hindawi.com
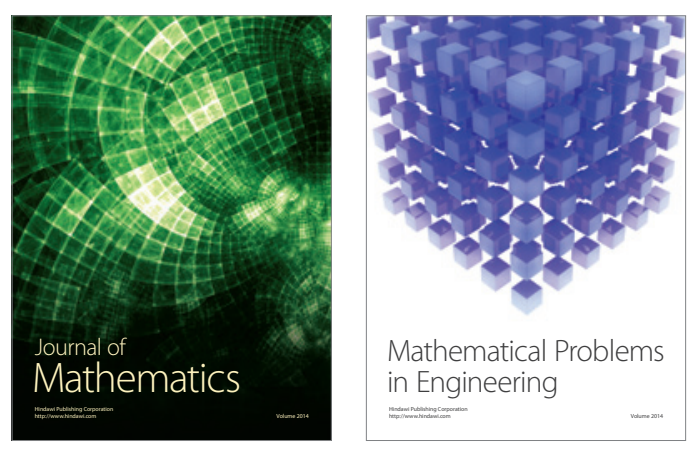

Mathematical Problems in Engineering
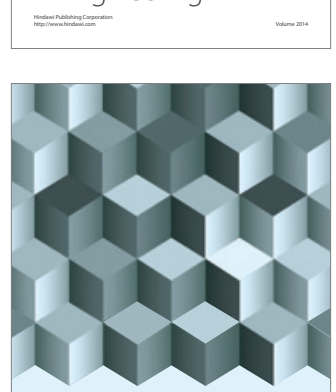

Journal of

Function Spaces
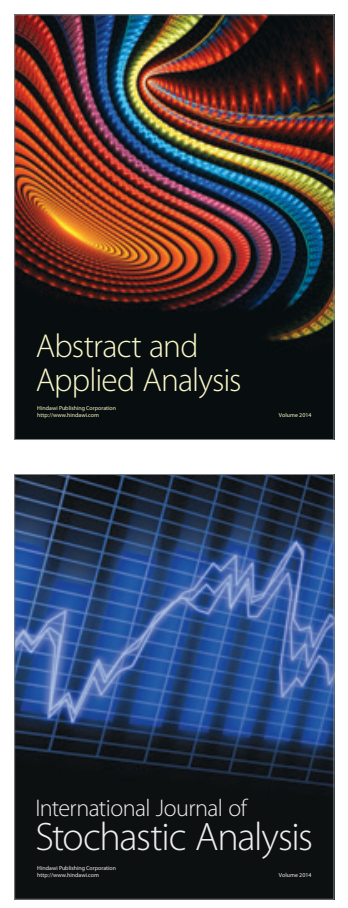

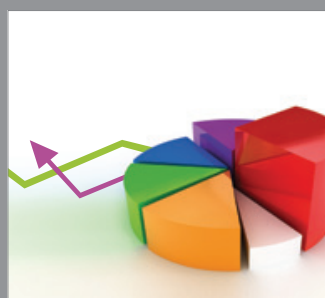

ournal of

Probability and Statistics

Promensencen
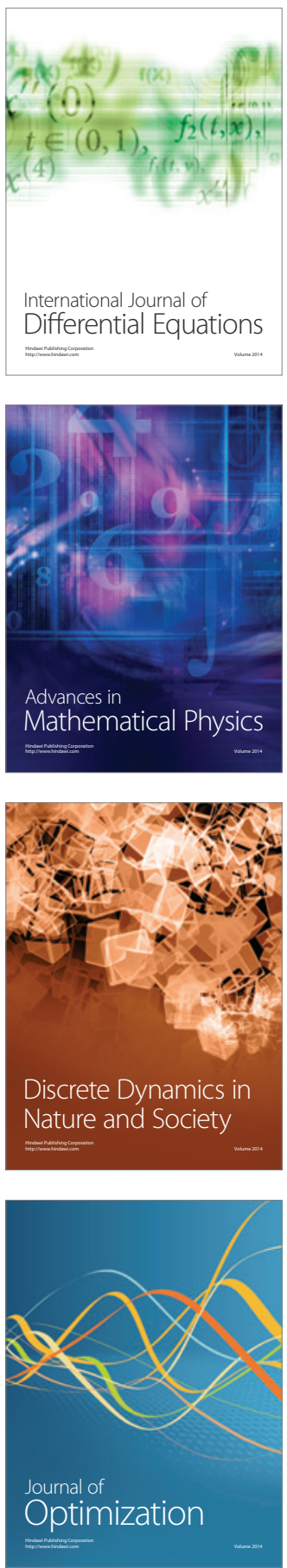